\title{
Noninvasive Imaging of Colitis Using Multispectral Optoacoustic Tomography
}

\author{
Neal Bhutiani ${ }^{1}$, William E. Grizzle ${ }^{2}$, Susan Galandiuk ${ }^{1}$, Denis Otali ${ }^{2}$, Gerald W. Dryden ${ }^{1}$, Nejat K. Egilmez ${ }^{3}$, \\ and Lacey R. McNally \\ ${ }^{I}$ Department of Surgery, University of Louisville, Louisville, Kentucky; ${ }^{2}$ Department of Pathology, University of Alabama \\ Birmingham, Birmingham, Alabama; ${ }^{3}$ Department of Microbiology and Immunology, University of Louisville, Louisville, Kentucky; \\ and ${ }^{4}$ Department of Cancer Biology, Wake Forest Baptist Comprehensive Cancer Center, Winston-Salem, North Carolina
}

\begin{abstract}
Currently, several noninvasive modalities, including MRI and PET, are being investigated to identify early intestinal inflammation, longitudinally monitor disease status, or detect dysplastic changes in patients with inflammatory bowel disease. Here, we assess the applicability and utility of multispectral optoacoustic tomography (MSOT) in evaluating the presence and severity of colitis. Methods: C57B/6 mice were untreated or treated with Bacteroides fragilis and antibiotic-mediated depletion of intestinal flora to initiate colitis. Mice were imaged using MSOT to detect intestinal inflammation. Intestinal inflammation identified with MSOT was also confirmed using both colonoscopy and histology. Results: Mice with bacterial colitis demonstrated a temporally associated increase in mesenteric and colonic vascularity with an increase in mean signal intensity of oxygenated hemoglobin $(P=0.004)$ by MSOT $2 \mathrm{~d}$ after inoculation. These findings were significantly more prominent $7 \mathrm{~d}$ after inoculation, with increased mean signal intensity of oxygenated hemoglobin $(P=0.0002)$ and the development of punctate vascular lesions on the colonic surface, which corresponded to changes observed on colonoscopy as well as histology. Conclusion: With improvements in depth of tissue penetration, MSOT may hold potential as a sensitive, accurate, noninvasive imaging tool in the evaluation of patients with inflammatory bowel disease.
\end{abstract}

Key Words: animal imaging; gastrointestinal; molecular imaging; colitis; inflammatory bowel disease; multispectral optoacoustic tomography; oxygenated hemoglobin

J Nucl Med 2017; 58:1009-1012

DOI: 10.2967/jnumed.116.184705

I nflammatory bowel disease (IBD) has grown increasingly prevalent over the last half century, with an annual incidence of approximately 250-300 per 100,000 persons in North America (1). Diagnosis usually involves a combination of factors identified in a patient's history and on physical examination, stool studies, and ultimately, colonoscopy with multiple biopsies (2). Furthermore, given the increased risk of colorectal cancer in patients with IBD, current practice guidelines recommend annual screening colonoscopies $8-10$ y after onset of disease to assess for dysplastic changes and status of disease

Received Sep. 23, 2016; revision accepted Nov. 17, 2016.

For correspondence or reprints contact: Lacey R. McNally, Department of Cancer Biology, Wake Forest Baptist Comprehensive Cancer Center, 1 Medical Center Blvd., Nutrition Building Rm. 443, Winston-Salem, NC 27157. E-mail: lacey_mcnally@hotmail.com

Published online Dec. 1, 2016.

COPYRIGHT (C) 2017 by the Society of Nuclear Medicine and Molecular Imaging.
(3). In addition to being especially uncomfortable for patients with IBD, colonoscopy represents an invasive procedure that carries risk from both sedation or anesthesia and colonic perforation. To date, no noninvasive imaging modalities have been developed that accurately identify early inflammatory changes in patients' small bowel and colon, that can be used longitudinally to monitor the status of disease, or that detect changes concerning for malignancy.

Multispectral optoacoustic tomography (MSOT) represents a novel modality that detects sound waves resulting from specific molecular excitation by light. Specifically, laser stimulation of tissues results in generation and emission of ultrasound waves. Ultrasound waves demonstrate a significantly decreased scatter compared with light waves before reaching the detecting transducer (4). Thus, MSOT retains speed and sensitivity while having an improved signal-to-noise ratio compared with conventional radiographic imaging modalities (e.g., CT and MRI (4-13)). To date, MSOT has demonstrated a resolution at $78.9 \mu \mathrm{m}(5,6)$. Furthermore, it can detect changes in tissue architecture and the presence of oxygenated and deoxygenated hemoglobin (5-10), permitting evaluation of changes in structure and vascularity, common in IBD. Recently, MSOT instrumentation has evolved and includes the potential for combination of detecting both optoacoustic and ultrasound signals, which increases its radiologic capabilities. Currently, MSOT has been used to effectively image tumor xenografts (5-9) as well as several orthotopic tumor models, including pancreatic adenocarcinoma $(5,7)$, but not to specifically assess inflammatory or dysplastic changes in the bowel in murine models.

Colonization with enterotoxic Bacteroides fragilis (ETBF), a pathogenic variant of a human intestinal commensal organism, has been implicated in the pathogenesis of IBD (14-15). Indeed, wild-type C57B/6 mice inoculated with ETBF after antibiotic-mediated depletion of intestinal flora can either initiate colitis or worsen susceptibility to colitis induced by other means $(14,15)$. Like colitis observed in IBD patients, the inflammation persists over time and comprises both local and systemic components. Here, we use this model of ETBF-induced colitis in $\mathrm{C} 57 \mathrm{~B} / 6$ mice to evaluate the ability of MSOT to detect intestinal and colonic inflammation.

\section{MATERIALS AND METHODS}

\section{Mice}

C57B/6 mice (Jackson Labs) were bred in accordance with University of Louisville (UofL) Institutional Animal Care and Use Committee (IACUC) guidelines. Six- to 7-wk-old mice of both genders were used for all experiments. A total of 9 mice were used for experiments. All experiments were conducted in accordance with UofL IACUC guidelines. Supplemental Figure 1 (supplemental materials are available at http://jnm. snmjournals.org) shows an experimental schematic. 
TABLE 1

Murine Endoscopic Index of Colitis Severity for Objective Quantification of Colonoscopic Findings

\begin{tabular}{|c|c|c|c|c|c|}
\hline \multirow[b]{2}{*}{ Finding } & \multicolumn{5}{|c|}{ Murine endoscopic index of colitis severity } \\
\hline & 0 & 1 & 2 & 3 & Total \\
\hline Thickening of the colon & Transparent & Moderate & Marked & Nontransparent & $0-3$ \\
\hline Changes of the vascular pattern & Normal & Moderate & Marked & Bleeding & $0-3$ \\
\hline Fibrin visible & None & Little & Marked & Extreme & $0-3$ \\
\hline Granularity of the mucosal surface & None & Moderate & Marked & Extreme & $0-3$ \\
\hline Stool consistency & Normal + solid & Still shaped & Unshaped & Spread & $0-3$ \\
\hline
\end{tabular}

Changes in colon thickness, vasculature, fibrin deposition, mucosal granularity, and stool consistence were all noted and assigned a score based on severity. These scores were summed to yield an overall colitis severity score. Table was modified from Becker et al. (16).

Bacteria. ETBF strain 86-5443-2-2 was cultured under anaerobic conditions on brain-heart infusion + clindamycin agar plates. Plates were streaked with bacterial stock stored in glycerol and subsequently incubated under anaerobic conditions at $37^{\circ} \mathrm{C}$. After $48 \mathrm{~h}$, bacteria were harvested and suspended in liquid brain-heart infusion + clindamycin broth before being incubated under anaerobic conditions for another $24 \mathrm{~h}$.

\section{Induction of Colitis}

To deplete enteric pathogen load and facilitate ETBF colonization, mice were administered clindamycin $(0.1 \mathrm{~g} / \mathrm{L})$ and streptomycin $(5 \mathrm{~g} / \mathrm{L})$ dissolved in drinking water for $4 \mathrm{~d}$ before bacterial inoculation. Approximately $1 \times 10^{8}$ bacteria suspended in $200 \mu \mathrm{L}$ of phosphatebuffered saline were then administered via oral gavage into the gastrointestinal tract of these mice. Mice that received neither antibiotics nor bacteria were used as controls. All mice were fed casein-based, low-anthocyanin chow ad libitum (TekLad 2920X; Envigo) and monitored closely for signs of dehydration and provided daily with electrolyte-rich gel supplements.

\section{Imaging and Statistical Comparison}

MSOT imaging was performed as previously described (6). Briefly, mice were anesthetized with $1.6 \%$ isoflurane inhalant delivered in $0.8 \mathrm{~L}$ of medical air and $0.1 \mathrm{~L} \mathrm{of}_{2}$, then depilated using a combination of shaving and application of Nair with aloe (Church \& Dwight Co.), which was removed with moist gauze. Mice were subsequently placed prone and imaged from the superior thorax to inferior pelvis with the MSOT system InVision 256TF (iThera Medical) using wavelengths of $680,710,730,740,760,770,780,800,850$, and $900 \mathrm{~nm}$ with 25 averages per wavelength and an acquisition time of $10 \mu \mathrm{s}$ per frame. Accurate subject positioning within the MSOT device was ensured using the device's image preview function. Mice were evaluated before bacterial inoculation (untreated normal control), $2 \mathrm{~d}$ after bacterial inoculation, and $7 \mathrm{~d}$ after bacteria inoculation. Images were reconstructed via backprojection with $75-\mu \mathrm{m}$ resolution. MSOT values for oxygenated and deoxygenated hemoglobin were determined using MSOT imaging software (ViewMSOT 3.5) and compared using linear regression (JMP software; JMP, SAS Institute Inc.). Region-of-interest analysis was used to determine oxy- and deoxy-hemoglobin separately (6) within the colon using a $3.5-\mathrm{mm}^{2}$ ellipse on 4 regions of the mouse colon per time point. The mean oxygenated hemoglobin signal intensity among the 4 regions was determined and compared using a 1-way ANOVA test with Tukey honest significant difference with JMP software. Differences were considered significant for a $P$ value of less than 0.05 .

\section{Colonoscopy and Statistical Comparison}

Mice were injected intraperitoneally with $0.1 \mathrm{~mL} / 20 \mathrm{~g}$ mouse weight of a ketaminexylazine cocktail $(87.5 \mathrm{mg} / \mathrm{kg}$ ketamine + $12.5 \mathrm{mg} / \mathrm{kg}$ xylazine). After an adequate level of anesthesia was achieved, an 8-French pediatric cystoscope was introduced into the mouse anus and advanced proximally until the scope could no longer be advanced $(15,16)$. The colonoscope was then withdrawn slowly. The Murine Endoscopic Index of Colitis Severity was used to quantify colitis severity (Table 1). Colitis severity score was compared among and between groups using 1-way ANOVA with Tukey honest significant difference. Correlation between mean 

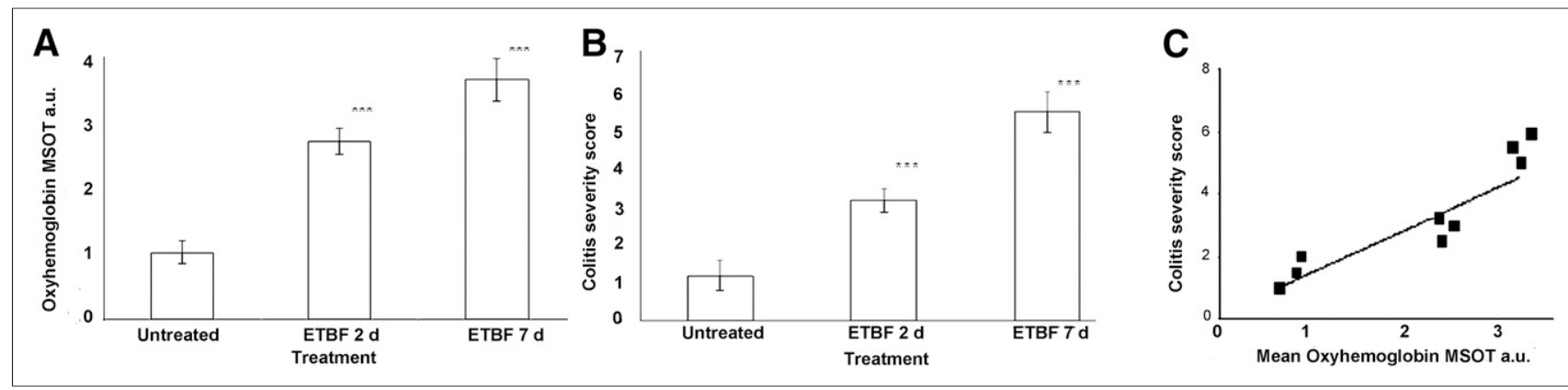

FIGURE 2. Mean MSOT signal intensity for oxyhemoglobin correlates with colitis severity score. Region of intensity measurements acquired for MSOT images were correlated to colitis severity score determined from colonoscopy images. (A) Mean signal intensity of oxyhemoglobin for each group (control, $2 \mathrm{~d}$ after ETBF inoculation, and $7 \mathrm{~d}$ after ETBF inoculation). ${ }^{\star \star \star} P<0.001$. (B) Average colitis severity score for each group. ${ }^{\star \star \star} P<0.001$. Error bars represent SD. (C) Correlation between mean signal intensity of oxyhemoglobin and mean colitis severity score for the 3 mice assessed using both MSOT and colonoscopy at all 3 time points $(r=0.82, P=0.013)$. a.u. $=$ arbitrary units.

MSOT oxygenated and deoxygenated hemoglobin intensity and colitis severity score for mice evaluated with both MSOT and colonoscopy at all 3 time points was assessed using Pearson correlation. Statistical analyses were performed using JMP software. Differences were considered significant for a $P$ value of less than 0.05 .

\section{Histology}

After colonoscopy, mice were euthanized. The colon was resected in its entirety and flushed twice with phosphate-buffered saline to evacuate residual stool. Colons were then bisected, fixed in $10 \%$ neutral-buffered formalin, and embedded in paraffin. Sections were cut at $6-\mu \mathrm{m}$ thickness and stained using hematoxylin and eosin. Samples were masked to the pathologist on histologic analysis by a diagnostic pathologist at the University of Alabama, Birmingham, Department of Pathology.

\section{RESULTS}

Mice treated with ETBF showed a mild increase in vascularity $2 \mathrm{~d}$ after bacterial inoculation (Figs. 1 and 2; Supplemental Fig. 2), with an increase in mean signal intensity of oxygenated hemoglobin compared with untreated mice (1.150 vs. 2.716 MSOT arbitrary units compared with untreated mice; $P=0.004)$. These findings were more prominent $7 \mathrm{~d}$ after inoculation, with increased mean signal intensity of oxygenated hemoglobin (1.150 vs. 2.716 vs. 3.422 MSOT arbitrary units for controls vs. $2 \mathrm{~d}$ after ETBF vs. $7 \mathrm{~d}$ after ETBF, $P=0.0002$ ) and the development of punctate lesions on the colonic surface (Fig. 1C). Deoxygenated hemoglobin signal obtained via MSOT remained similar in all mice evaluated regardless of treatment or time point.

The findings on MSOT correlated well with colonoscopic findings ( $r=0.82, P=0.013)$. Compared with untreated controls, mice at $2 \mathrm{~d}$ after ETBF inoculation demonstrated an increased colitis score (1.5 vs. 2.5 ), with mild colonic blunting and slightly deformable stool (Figs. 1D and 1E). At $7 \mathrm{~d}$ after ETBF inoculation, mice displayed colonic blunting, vascular aneurysms, fibrin coats, patchy granularity, and deeply deformable stool (Fig. 1F), yielding an average colitis score of 5.5 (Figs. 1 and 2B).

Additionally, findings on MSOT and colonoscopy corresponded well with masked histologic analysis. Mice showed evidence of increasing inflammatory cell infiltrate and architectural distortion at both 2 and $7 \mathrm{~d}$ after ETBF inoculation compared with controls (Fig. 3). A similar progression was also evident comparing mice 2 and $7 \mathrm{~d}$ after ETBF inoculation. Findings were consistent across all mice in each group.

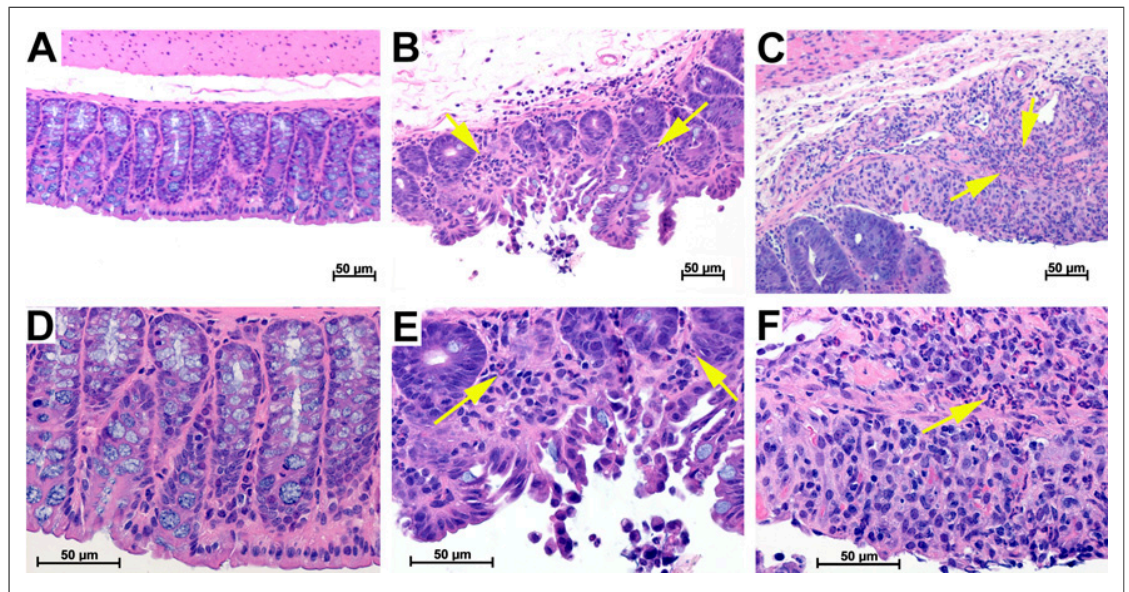

FIGURE 3. Histology findings demonstrate inflammatory changes consistent with colitis. Hematoxylin and eosin analysis of mouse colon demonstrated no evidence of inflammatory cell infiltrate in control mice $(A$ and $D)$ and progressively increasing polymorphonuclear leukocyte infiltrate $2 \mathrm{~d}(\mathrm{~B}$ and $\mathrm{E})$ and $7 \mathrm{~d}(\mathrm{C}$ and $\mathrm{F})$ after ETBF inoculation as indicated by arrows. Histology images shown in $\mathrm{C}$ and $\mathrm{F}$ are from mouse represented in Figure 1.

\section{DISCUSSION}

Our findings demonstrate that MSOT findings of hypervascularity and elevated levels of oxyhemoglobin are associated with inflammatory changes in the colon as well as inflammatory cell infiltrate evidenced on histology; this was associated with mild inflammatory changes on histology that were minimally detectable on colonoscopy in the same mice.

Clinically, MSOT offers multiple advantages over all current imaging and monitoring modalities including higher resolution without requiring exogenous contrast agents. Because quantification of oxygenated hemoglobin correlates with vascular changes seen on colonoscopy and inflammatory changes seen on histology, MSOT can provide an objective assessment tool that can be used to monitor the severity and progression. 
Additionally, given that polyposis and tumor growth are associated with local neo-/hypervascularization, MSOT can be used to monitor IBD patients for tumorigenesis, with colonoscopy used only if concerning features are identified on imaging studies.

Use of MSOT for diagnosis and monitoring of IBD has the potential to significantly affect disease prognosis. The MSOT apparatus for use in human subjects consists of a handheld probe similar to bedside ultrasound $(5,10,17,18)$. Improvements in depth of tissue penetration of optoacoustic imaging beyond $1 \mathrm{~cm}$ have been demonstrated in humans using the clinical MSOT system, Acuity, with up to a $5-\mathrm{cm}$ depth achieved $(17,18)$. Therefore, MSOT could be used to provide noninvasive screening for IBD in patients at high risk for developing the disease because of factors such as family history and ethnicity, thereby enabling earlier detection of disease and commencement of therapy to prevent progression. Additionally, small changes detected on MSOT that may predict clinical worsening of disease (e.g., increased vascularity, development of small polyps) can prompt alteration of medical therapy or more timely colonoscopy and, if necessary, surgery to prevent clinical worsening of disease or delayed detection of malignancy and risk of local invasion or metastasis. Conversely, intervals between invasive monitoring with colonoscopy can be increased in patients lacking significant changes in colonic and mesenteric vascularity and polyposis on MSOT over time. This would decrease monitoring cost as well as procedural risks and discomfort for IBD patients.

\section{CONCLUSION}

MSOT represents a noninvasive diagnostic modality that effectively identifies colitis in a murine model. Its diagnostic accuracy is at least equivalent to current standards of colonoscopy and tissue histology and surpasses that of conventional imaging modalities. With improvements in deep-tissue penetration, these factors, together with MSOT's detection of oxygenated and deoxygenated hemoglobin as endogenous contrast agents and correlation of signal intensity with colitis severity, would allow MSOT to serve as a viable modality for diagnosis and monitoring of patients with IBD for both progression of disease and development of neoplastic intestinal lesions.

\section{DISCLOSURE}

This work was supported by NIH grants R01CA205941 (Lacey R. McNally), R01EB020125 (Lacey R. McNally), R01CA100656
(Nejat K. Egilmez), and P30CA013148 (William E. Grizzle). No other potential conflict of interest relevant to this article was reported.

\section{REFERENCES}

1. Molodecky NA, Soon IS, Rabi DM, et al. Increasing incidence and prevalence of the inflammatory bowel diseases with time, based on systematic review. Gastroenterology. 2012;142:46-54.e42.

2. Tontini GE, Vecchi M, Pastorelli L, et al. Differential diagnosis in inflammatory bowel disease colitis: state of the art and future perspectives. World J Gastroenterol. 2015;21:21-46.

3. Mattar MC, Lough D, Pishvaian MJ, Charabaty A. Current management of inflammatory bowel disease and colorectal cancer. Gastrointest Cancer Res. 2011;4:53-61.

4. Ntziachristos V, Razansky D. Optical and opto-acoustic imaging. Recent Results Cancer Res. 2013;187:133-150.

5. McNally LR, Mezera M, Morgan DE, et al. Current and emerging clinical applications of multispectral optoacoustic tomography (MSOT) in oncology. Clin Cancer Res. 2016;22:3432-3439.

6. Bhutiani N, Kimbrough CW, Burton N, et al. Detection of microspheres in vivo using multispectral optoacoustic tomography. Biotech Histochem. In press.

7. Kimbrough CW, Khanal A, Zeiderman M, et al. Targeting acidity in pancreatic adenocarcinoma: multispectral optoacoustic tomography detects $\mathrm{pH}$-low insertion peptide probes in vivo. Clin Cancer Res. 2015;21:4576-4585.

8. Burton NC, Patel M, Morscher S, et al. Multispectral opto-acoustic tomography (MSOT) of the brain and glioblastoma characterization. Neuroimage. 2013;65:522528 .

9. Cao J, Campbell J, Liu L, Mason RP, Lippert AR. In vivo chemiluminescent imaging agents for nitroreductase and tissue oxygenation. Anal Chem. 2016;88:49955002.

10. Waldner MJ, Knieling F, Egger C, et al. Multispectral optoacoustic tomography in Crohn's disease: noninvasive imaging of disease activity. Gastroenterology. 2016;151:238-240.

11. Razansky D, Buehler A, Ntziachristos V. Volumetric real-time multispectral optoacoustic tomography of biomarkers. Nat Protoc. 2011;6:1121-1129.

12. Ma R, Taruttis A, Ntziachristos V, Razansky D. Multispectral optoacoustic tomography (MSOT) scanner for whole-body small animal imaging. Opt Express. 2009; 17:21414-21426.

13. Dietrich O, Raya JG, Reeder SB, Reiser MF, Schoenberg SO. Measurement of signal-to-noise ratios in MR images: influence of multichannel coils, parallel imaging, and reconstruction filters. J Magn Reson Imaging. 2007;26:375-385.

14. Rabizadeh S, Rhee KJ, Wu S, et al. Enterotoxigenic bacteroides fragilis: a potential instigator of colitis. Inflamm Bowel Dis. 2007;13:1475-1483.

15. Rhee KJ, Wu S, Wu X, et al. Induction of persistent colitis by a human commensal, enterotoxigenic bacteroides fragilis, in wild-type C57BL/6 mice. Infect Immun. 2009;77:1708-1718.

16. Becker C, Fantini MC, Neurath MF. High resolution colonoscopy in live mice. Nat Protoc. 2006;1:2900-2904

17. Buehler A, Kacprowicz M, Taruttis A, Ntziachristos V. Real-time handheld multispectral optoacoustic imaging. Opt Lett. 2013;38:1404-1406.

18. Stoffels I, Morscher S, Helfrich I, et al. Metastatic status of sentinel lymph nodes in melanoma determined noninvasively with multispectral optoacoustic imaging. Sci Transl Med. 2015;7:317ra199. 